\title{
ABOUT THE PRICING EQUATION IN FINANCE
}

\author{
Stéphane CRÉPEY \\ University of Evry, France \\ stephane.crepey@univ-evry.fr \\ AMAMEF at Vienna University of Technology \\ 17-22 September 2007
}


We derive the pricing equation of a general (American or Game) Contingent Claim in the set-up of a rather generic Markovian factor process.

As an aside we establish the convergence of stable, monotone and consistent approximation schemes to the pricing function (solution to the pricing equation). 


\section{Model}

We first introduce a flexible Markovian model $\mathcal{X}=(X, N)$ made of a jump diffusion $X$ interacting with a pure jump process $N$ (which in the simplest case reduces to a Markov chain in continuous time).

The state space is $\mathcal{E}=[0, T] \times \mathbb{R}^{d} \times I$ with $I=\{1, \cdots, k\}$, where $T>0$ is a finite horizon and $d$ and $k$ denote positive integers.

Note that a function $u=u(t, x, i)$ on $[0, T] \times \mathbb{R}^{d} \times I$ may equivalently be considered as a system $u=\left(u^{i}\right)_{i \in I}$ of functions $u^{i}=u^{i}(t, x)$ on $[0, T] \times \mathbb{R}^{d}$. Likewise we denote $u(t, x, i, j)$, or $u^{i, j}(t, x)$ for a function $u$ of $(t, x, i, j)$.

Let us be given a stochastic basis $(\Omega, \mathbb{F}, \mathbb{P})$ on $[0, T]$ endowed with a $d$-dimensional Brownian motion $B$ and an integer-valued random measure $\chi$. Our model consists of an $\mathbb{R}^{d} \times I$-valued Markov càdlàg process $\mathcal{X}=(X, N)$ on $[0, T]$ with initial condition $(x, i)$ at time 0 , such that the $\mathbb{R}^{d}$-valued process 


\section{$X$ satisfies:}

$$
d X_{t}=b\left(t, X_{t}, N_{t}\right) d t+\sigma\left(t, X_{t}, N_{t}\right) d B_{t}+\int_{\mathbb{R}^{d}} \delta\left(t, X_{t-}, N_{t-}, y\right) \widetilde{\chi}(d y, d t)
$$

where:

- the compensated jump measure $\tilde{\chi}$ of $\chi$ is given by

$$
\widetilde{\chi}(d t, d y)=\chi(d t, d y)-f\left(t, X_{t}, N_{t}, y\right) m(d y) d t
$$

so that the intensity measure of the jumps of $X$ is given by $f\left(t, X_{t}, N_{t}, y\right), y \in \mathbb{R}^{d}$;

- the intensity measure of the $I$-valued pure jump process $N$ is given by $\mathbb{1}_{\left\{N_{t} \neq j\right\}} \lambda\left(t, X_{t}, N_{t}, j\right), j \in I$.

In these equations $m(d y)$ is a jump probability measure on $\mathbb{R}^{d} \backslash\{0\}$, and all the coefficients are Borel-measurable functions such that:

- the $\sigma^{i}(t, x)$ are $d$-dimensional dispersion matrices, with related covariance 
matrices $a^{i}(t, x)=\sigma^{i}(t, x) \sigma^{i}(t, x)^{\top}$;

- the $b^{i}(t, x)$ are $d$-dimensional drift vector coefficients;

- the intensity functions $f^{i}(t, x, y)$ are bounded, and the jump size functions $\delta^{i}(t, x, y)$ are bounded w.r.t $y$ at fixed $(t, x)$, locally uniformly in $(t, x)$;

- the $\left[\lambda^{i, j}(t, x)\right]_{i, j \in I}$ are intensity matrices such that the functions $\lambda^{i, j}(t, x)$ are non-negative and bounded for $i \neq j$, and $\lambda^{i, i}(t, x)=-\sum_{j \in I \backslash\{i\}} \lambda^{i, j}(t, x)$.

This model admits versatile applications in financial modeling. 


\section{Main Result}

Let us define the following linear operator $\mathcal{G}$ acting on regular functions $u=u^{i}(t, x)$ for $(t, x, i) \in \mathcal{E}$, and where $\partial u$ (resp. $\mathcal{H} u$ ) denotes the row-gradient (resp. Hessian) of $u(t, x, i)=u^{i}(t, x)$ with respect to $x$ :

$$
\begin{aligned}
& \mathcal{G} u^{i}(t, x)=\partial_{t} u^{i}(t, x)+\frac{1}{2} \operatorname{Tr}\left[a^{i}(t, x) \mathcal{H} u^{i}(t, x)\right] \\
& \quad+\partial u^{i}(t, x)\left(b^{i}(t, x)-\int_{\mathbb{R}^{d}} \delta^{i}(t, x, y) f^{i}(t, x, y) m(d y)\right)+\mathcal{I} u^{i}(t, x)
\end{aligned}
$$

where

$$
\mathcal{I} u^{i}(t, x)=\int_{\mathbb{R}^{d}}\left(u^{i}\left(t, x+\delta^{i}(t, x, y)\right)-u^{i}(t, x)\right) f^{i}(t, x, y) m(d y) .
$$

Let us further be given a system of real-valued continuous cost functions with polynomial growth in $x$, namely a running cost function $g^{i}(t, x, u, z, r)$ (where $\left.(u, z, r) \in \mathbb{R}^{k} \times \mathbb{R}^{1 \otimes d} \times \mathbb{R}\right)$, a terminal cost function $\Psi^{i}(x)$, and lower and upper cost functions $\ell^{i}(t, x)$ and $h^{i}(t, x)$, such that: 
- $g$ is Lipschitz continuous with respect to $(u, z, r)$ and non-decreasing with respect to $r$;

- $\ell \leq h$ and $\ell(T, \cdot) \leq \Psi \leq h(T, \cdot)$;

- $\ell=\phi \vee c$ for a 'regular' function $\phi$ on $\mathcal{E}$ and for a constant $c \in \mathbb{R} \cup\{-\infty\}$.

We denote by $(\mathcal{V} 2)$ the following variational inequality with double obstacle problem (reaction-diffusion system):

$$
\begin{gathered}
\max \left(\operatorname { m i n } \left(-\mathcal{G} u^{i}(t, x)-g^{i}\left(t, x, u(t, x),(\partial u \sigma)^{i}(t, x), \mathcal{I} u^{i}(t, x)\right)\right.\right. \\
\left.\left.u^{i}(t, x)-\ell^{i}(t, x)\right), u^{i}(t, x)-h^{i}(t, x)\right)=0
\end{gathered}
$$

on $\{t<T\}$ supplemented by the terminal condition $\Psi$ (the terminal cost function) at $T$.

Note that as opposed to the set-up of Becherer and Schweizer [7] where linear reaction-diffusion systems of parabolic equations are considered in a diffusion model with regimes (but without jumps in $X$ ), here, due to the presence of the obstacles in the problem and of the jumps in $X$, the related reaction-diffusion 
system $(\mathcal{V} 2)$ typically does not have classic solutions.

By a solution to $(\mathcal{V} 2)$, we thus mean a viscosity solution with polynomial growth in $x$ to $(\mathcal{V} 2)$, adapting the general definitions of viscosity solutions for nonlinear PDEs (see [14, 19]) to (finite activity) jumps and systems of PIDEs as in [1, 22, 4, 13]. A complementary weak Sobolev solutions approach is dealt with in Crépey-Matoussi [17].

The notions of stable, monotone and consistent approximation schemes below were originally introduced for nonlinear PDEs by Barles and Souganidis [6], and further extended to PIDEs by Briani, La Chioma and Natalini [13]).

Theorem 2.1 'Under suitable conditions:'

(i) (V2) admits a unique solution $u$. Moreover, $u$ admits a probabilistic interpretation (Feynman-Kac formula) in terms of a related control problem (doubly reflected BSDE).

(ii) Any stable, monotone and consistent approximation scheme $\left(u_{h}\right)$ converges locally uniformly to $u$ as $h \rightarrow 0$. 
Remark 2.1 (i) In part (i), existence and representation are obtained by BSDE techniques (see Crépey and Matoussi [18], Crépey [16]) whereas uniqueness results from typical viscosity arguments (cf. in particular Barles et al. [4]). (ii) Part (ii) extends to models with regimes (whence systems of PIDEs) the convergence results of [6, 13]. 


\section{Application in Finance}

We now specify the above set-up to risk-neutral pricing problems in finance. In this case:

- the function $g$ is typically of the form:

$$
g^{i}(t, x, u, z, r)=c^{i}(t, x)-\mu^{i}(t, x) u^{i}+\sum_{j \in I \backslash\{i\}}\left(u^{j}-u^{i}\right) \lambda^{i, j}(t, x),
$$

for dividend and interest-rate related functions $c$ and $\mu$ (or dividends and interest-rates adjusted for credit spread, in a more general context of defaultable contingent claims);

- $\Psi\left(\mathcal{X}_{T}\right)$ corresponds to a terminal payoff that is paid by the issuer to the holder at time $T$ if the contract was not exercised before $T$;

- $\ell\left(t, \mathcal{X}_{t}\right)$, resp. $h\left(t, \mathcal{X}_{t}\right)$, corresponds to a lower, resp. upper payoff that is paid by the issuer to the holder of the claim in the event of early termination of the contract at the initiative of the holder, resp. issuer.

The contingent claims under consideration are thus general Game Contingent 
Claims (see [8, 9, 10, 11]), covering American Claims (and European Claims) as special cases. From the point of view of the financial interpretation, the components of $\mathcal{X}$ are observable factors.

Along with this factor process $\mathcal{X}$, we consider a primary market composed of the savings account and of $d+k$ primary risky assets with locally bounded semimartingale price process $\mathcal{Y}=\left(Y^{1}, \cdots, Y^{d+k}\right)^{\top}$. The discount factor $\beta$, that is, the inverse of the savings account, is defined as $\beta_{t}=\exp \left(-\int_{0}^{t} \mu_{u} d u\right)$ (so $\mu$ can be interpreted as a short-term interest rate process). For simplicity of presentation we assume no dividends on the primary risky assets.

Consistently with arbitrage requirements on the underlying market, we postulate that $\beta \mathcal{Y}$ is a $\mathbb{P}$ - local martingale, where the probability measure $\mathbb{P}$ is equivalent to the objective probability measure on the underlying market.

The factor process $\mathcal{X}$ and the primary price process $\mathcal{Y}$ are typically intimately, though non-trivially, connected, as follows:

- Most factors are typically given as primary price processes. The components of $\mathcal{X}$ that are not included in $\mathcal{Y}$ (if any) are to be understood as simple factors that 
may be required to 'Markovianize' the payoffs of a game option (e.g., factors accounting for path dependence in the option's payoff and/or non-traded factors such as stochastic volatility in the dynamics of the assets underlying the option);

- Some of the primary price processes may not be needed as factors, but are used for hedging, so that condition (2) may be satisfied below.

Theorem 3.1 (i) (Arbitrage) The process $u\left(t, X_{t}, N_{t}\right), t \in[0, T]$ is an arbitrage price process for the Claim.

(ii) (Hedging; Work in progress) $u\left(0, \mathcal{X}_{0}\right)$ is the minimal initial wealth of an (issuer super-)hedge with $\mathbb{P}$ - local martingale residual cost process for the Claim. Denoting by $\widetilde{\nu}$ the compensated jump measure of $N$, let us assume further that

$$
\beta_{t} d\left(\begin{array}{c}
B_{t} \\
\widetilde{\nu}_{t}
\end{array}\right)=\Lambda_{t} d\left(\beta_{t} \mathcal{Y}_{t}\right), \quad t \in[0, T]
$$

for some $\mathbb{R}^{d+k \otimes d+k}$-valued predictable locally bounded process $\Lambda$. Then a 
hedge with $\mathbb{P}$ - local martingale residual cost process

$d n_{t}=\int_{\mathbb{R}^{d}}\left[u\left(t, X_{t-}+\delta\left(t, X_{t-}, N_{t-}, y\right), N_{t-}\right)-u\left(t, X_{t-}, N_{t-}\right)\right] \tilde{\chi}(d y, d t)$

is given by

$$
\begin{gathered}
\zeta_{t}=\left[\partial u \sigma\left(t, X_{t}, N_{t}\right), u\left(t, X_{t-}\right)-u\left(t, X_{t-}, N_{t-}\right)\right] \Lambda_{t}, \quad t \in[0, T] \\
\tau=\inf \left\{t \in[0, T] ; u\left(t, X_{t}, N_{t}\right) \in \mathcal{C}\right\} \wedge T
\end{gathered}
$$

where

$$
u(t, x)-u^{i}(t, x):=\left(u^{1}(t, x)-u^{i}(t, x), \cdots, u^{k}(t, x)-u^{i}(t, x)\right),
$$

and where

$$
\mathcal{C}:=\left\{(t, x, i) \in \mathcal{E} ; u^{i}(t, x)=h^{i}(t, x)\right\}
$$

is the Early Call Region.

Remark 3.1 (i) In the special case of a purely diffusive (no jumps nor regimes), 
complete market, with morally $\Lambda=\sigma^{-1}$, we recover the usual relation $\zeta_{t}=\partial u\left(t, X_{t}\right)$, ensuring a self-financing (super-)hedge to the issuer of the claim.

(ii) These results can be extended to defaultable Contingent Claims by passage to an equivalent fictitious default-free world with suitably modified discount factors and dividend processes (introducing a further defaultable primary asset to hedge default risk, if wished).

\section{References}

[1] Alvarez O., Tourin, A.: Viscosity solutions of nonlinear integro-differential equations. Annales de l'institut Henri Poincaré (C) Analyse non linéaire, 13 no. 3 (1996), p. 293-317.

[2] Amadori, A.L.: Nonlinear integro-differential evolution problems arising in option pricing: a viscosity solutions approach. J. Differential and Integral 
Equations 16(7), 787-811 (2003).

[3] Amadori, A.L.: The obstacle problem for nonlinear integro-differential operators arising in option pricing. Quaderno IAC Q21-000, (2000).

[4] Barles, G., Buckdahn, R. and Pardoux, E.: Backward Stochastic Differential Equatiions and Integral-Partial Differential Equations. Stochastics and Stochastics Reports, Vol. 60, pp. 57-83 (1997).

[5] Barles, G., Imbert, C.: Second-Order Elliptic Integro-Differential Equations: Viscosity Solutions' Theory Revisited. Annales de l'IHP, To Appear.

[6] G. Barles and P.E. Souganidis. Convergence of approximation schemes for fully nonlinear second order equations, Asymptotic Anal., (4), pp. 271-283, 1991.

[7] Becherer, D. and Schweizer, M: Classical Solutions to Reaction-Diffusion Systems for Hedging with Interacting Itô and Point Processes. Annals of Applied Probability, 15, 1111-1144, 2005. 
[8] Bielecki, T.R., Crépey, S., Jeanblanc, M. and Rutkowski, M.: Arbitrage pricing of defaultable game options with applications to convertible bonds. Forthcoming in Quantitative Finance.

[9] Bielecki, T.R., Crépey, S., Jeanblanc, M. and Rutkowski, M.: Valuation and hedging of defaultable game options in a hazard process model. Submitted, 2006.

[10] Bielecki, T.R., Crépey, S., Jeanblanc, M. and Rutkowski, M.: Defaultable options in a Markovian intensity model of credit risk. Forthcoming in Mathematical Finance.

[11] Bielecki, T.R., Crépey, S., Jeanblanc, M. and Rutkowski, M.: Convertible Bonds in a Defaultable Diffusion Model. Submitted.

[12] Bielecki, T.R., Crépey, S., Jeanblanc, M. and Rutkowski, M.: Valuation of basket credit derivatives in the credit migrations environment. Handbook of Financial Engineering, 2006.

[13] Briani M, La Chioma C and Natalini R. Convergence of numerical schemes 
for viscosity solutions to integro-differential degenerate parabolic problems arising in financial theory, Numer. Math., 2004, vol. 98, no4, pp. 607-646.

[14] M. Crandall, H. Ishii and P.-L. Lions. User's guide to viscosity solutions of second order partial differential equations, Bull. Amer. Math. Soc., 1992.

[15] Crépey, S.: About the Pricing Equation in Finance. Submitted.

[16] Crépey: Markovian Reflected and Doubly Reflected BSDEs in a Jump-Diffusion Setting with Regimes. Submitted.

[17] Crépey, S., Matoussi, A.: About the Greeking Equation in Finance. Work in preparation.

[18] Crépey, S., Matoussi, A.: Reflected and Doubly Reflected BSDEs with Jumps: A Priori Estimates and Comparison Principle. Submitted.

[19] W. Fleming and H. Soner. Controlled Markov processes and viscosity solutions, Second edition, Springer, 2006.

[20] Harraj. N., Ouknine. Y. and Turpin. I.. Double-barriers-reflected BSDEs with 
jumps and viscosity solutions of parabolic integrodifferential PDEs, Journal of Applied Mathematics and Stochastic Analysis (2005), 1, 37-53.

[21] E. R. Jakobsen and K. H. Karlsen.: A "maximum principle for semicontinuous functions" applicable to integro-partial differential equations. Nonlinear Differential Equations Appl., 13:137-165, 2006.

[22] E. Pardoux, F. Pradeilles, Z. Rao. Probabilistic interpretation of systems of semilinear PDEs. Annales de l'Institut Henri Poincaré, série Probabilités-Statistiques, 33, 467-490, 1997. 